\title{
Methylmalonic Acidemia - Cause of Recurrent Neonatal Death and It's Social Implications
}

\author{
Madhavi $\mathrm{V}^{1}$, Sharma $\mathrm{D}^{2}$, Murki $\mathrm{S}^{3}$, Pratap $\mathrm{T}^{4}$
}

\begin{abstract}
Mr and Mrs R, non-consanguineous couple had history of all their children during neonatal period. First two neonates were normal at birth, then presented with lethargy, vomiting and decreased acceptance of feeds. Both the babies expired after birth without being investigated for cause of death. The third neonate was investigated for inborn error of metabolism and found to be affected with methylmalonic acidemia. The couple departed away inspite of extensive counselling. In the fourth pregnancy antenatally fetus was diagnosed as carrier case of methylmalonic acidemia and short chain fatty acid oxidase deficiency. The fourth baby was normal and was in follow up till one year age.
\end{abstract}

\section{Introduction}

E stablishing diagnosis of Methylmalonic Acidemia (MMA) in the infant is important for providing accurate patient education, genetic counselling and estimation of recurrence risk and offering prenatal diagnosis. Parents can be provided with anticipatory guidelines regarding potential problems as well as management and therapeutic options. In this case report, we present a couple who had recurrent neonatal death because of MMA and when it was diagnosed in third infant, couple got separated and the carrier mother had normal carrier neonate with new partner.

\section{The Case}

Mr and Mrs R (fictitious names for patient confidentiality), third gravida, a non-consanguineous couple reported to our genetic clinic at 28 weeks of gestation with a history of three neonatal deaths. In her first pregnancy, she had uneventful antenatal period. A male baby was delivered at term gestation with birth weight of $2.8 \mathrm{~kg}$ through uneventful vaginal delivery. Baby cried immediately after birth with Apgar score at $1 \mathrm{~min}, 5 \mathrm{~min}$ and $10 \mathrm{~min}$ were 8,8 and 9 respectively. Baby was started on breast feeding soon after birth. On day three of life, baby developed lethargy, vomiting and decrease acceptance of feeds. The infant was admitted to NICU but infant expired on day five of life. No metabolic workup was done. She had similar history during her second pregnancy and the infant expired on day four. The cause of death in both the neonates was not investigated. However based on the history, we suspected metabolic disorder.
${ }^{1}$ Dr. Vasikarla, Madhavi, MBBS, MD, Fellow in Genetics Department of Genetics, ${ }^{2}$ Dr. Deepak Sharma, MD, DNB Neonatology (Student), Department of Neonatology, ${ }^{3}$ Dr. Srinivas Murki, MD, DM Neonatology, ${ }^{4}$ Dr. Tejo Pratap MD, DM Neonatology. All from Fernandez Hospital, Hyderabad, India.

\section{Address for correspondence:}

Dr. Srinivas Murki

E-mail: srinivasmurki2001@gmail.com

\section{How to cite}

Madhavi V, Sharma D, Murki S, Pratap T. Methylmalonic Acidemia - Cause of Recurrent Neonatal Death and It's Social Implications. J Nepal Paediatr Soc 2014;34(2):150-153.

doi: http://dx.doi.org/10.3126/jnps.v34i2.10577

This work is licensed under a Creative Commons Attribution 3.0 License.

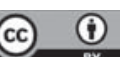

In the present pregnancy, metabolic screening and complete evaluation of the newborn was planned. She delivered a term AGA female baby with birth weight of $3 \mathrm{~kg}$ with Apgar scores of 8/9/9. On physical examination there was no gross dysmorphic features and infant was active and alert. The infant was started on breast feeds and in view of previous two sibling deaths, baby was kept under observation in the hospital. On day three, she became symptomatic with lethargy, decreased activity and vomiting. The infant was evaluated with sepsis screen, blood culture, electrolytes and blood sugar which were normal. ECHO and Neurosonogram were normal. Blood 
gas analysis revealed significant metabolic acidosis $(\mathrm{pH}$ of 7.01, Base deficit of -18) and increased anion gap. Baby was evaluated for inborn error of metabolism which showed diagnosis of Methylmalonic academia (very high values of methylmalonic acid, methyl citrate, proprionlygylcine, tiglylglycine, and 3 hydroxypropionic acid in urine). The infant expired on day 10 of life. In spite of extensive counselling that Methylmalonic Acidemia is an autosomal recessive disorder which has $25 \%$ recurrence risk in each pregnancy and prenatal diagnosis can be offered, the couple got divorced from each other. Mrs R had come to us in her fourth pregnancy with her second husband and requested for prenatal diagnosis. Prenatal diagnosis was done and it revealed marked elevation of butyryl carnitine and mild elevation of branched chain (c5: isovalaryl- / $\alpha$ methylbutryl) -carnitine, most compatible with benign short chain acyl-CoA dehydrogenase (SCAD) deficiency combined with the carrier state for MMA. Since it is a benign condition, Mrs.R continued pregnancy and delivered a healthy girl. The child remained normal during her first year of life during our follow up.

\section{Discussion}

Methylmalonic Acidemia (MMA) is an autosomal recessive inherited inborn error of metabolism with an incidence of around 1 in 50,000 live births. MMA result in increased level of acylcarnitine in blood and increased urinary excretion of methylmalonic acid. MMA group have a common defect in which ability to convert methylmalonyl-CoA into succinyl-CoA is defective. This defect can be either due to complete or partial deficiency methylmalonyl-CoA mutase apoenzyme or impaired synthesis of adenosylcobalamin, the cofactor for this enzyme or deficiency of the enzyme methylmalonyl-CoA epimerase. Methylmalonyl-CoA mutase is key enzyme for metabolism of four amino acid (isoleucine, methionine, threonine and valine) in the body ${ }^{1}$. The first case reports dates back to 1960 with varied manifestation like chronic metabolic acidosis, long chain ketonuria and hyperglycinenia ${ }^{2,3,4}$.

MMA has varied manifestation from neonatal period to adulthood ${ }^{5}$.

- $\quad$ Neonatal period: It present with signs overlapping with neonatal sepsis including lethargy, vomiting, truncal hypotonia, hypothermia, respiratory distress, severe ketoacidosis, hyperammonemia, neutropenia, and thrombocytopenia, neonatal death. In our case all the infants had symptoms in neonatal periods and had similar features.

- Infantile period: This has two subtype on the basis of response to Vitamin B12.

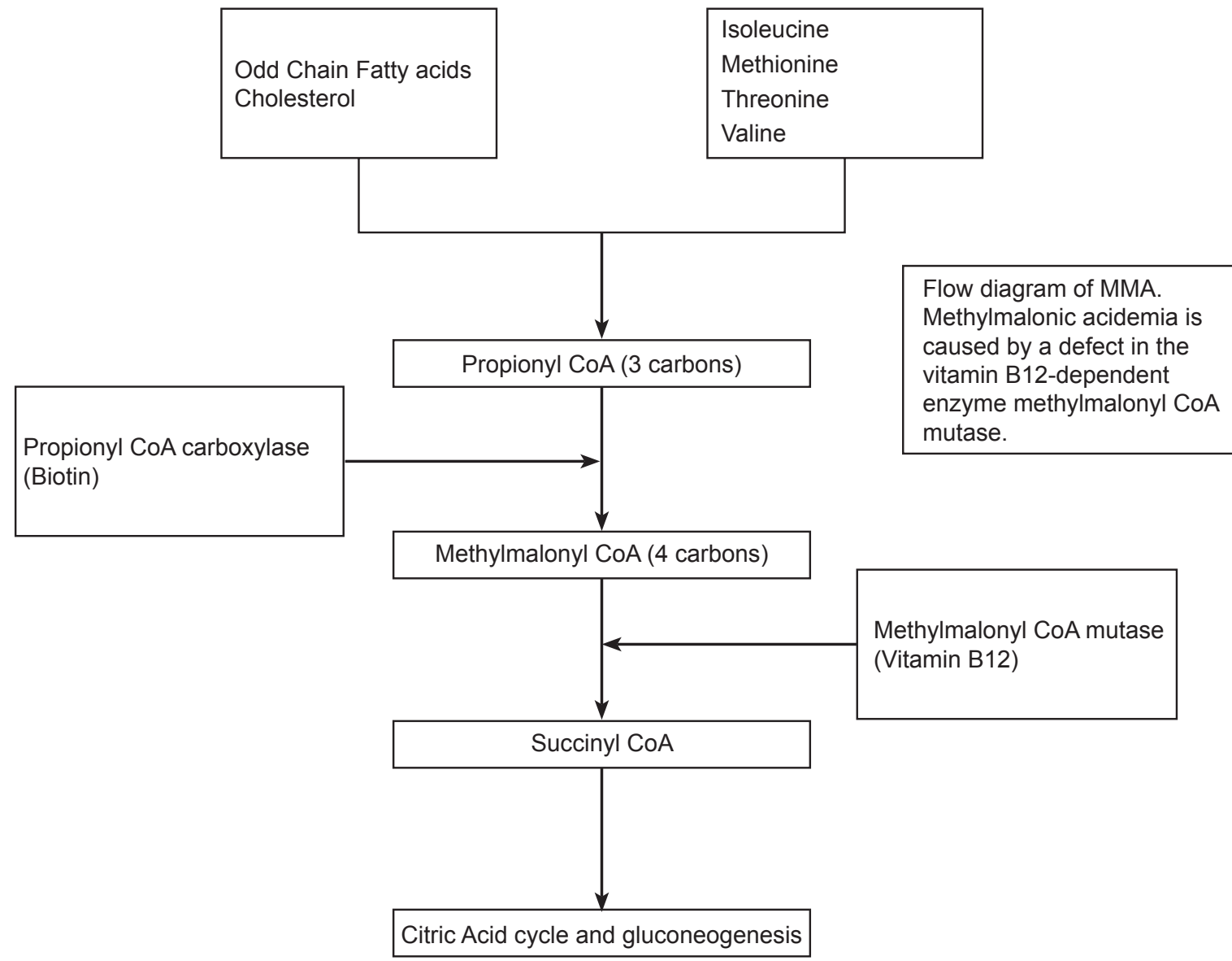


- Non-B12-responsive phenotype: This is the most common variant with infants who will be normal at birth but develop lethargy, vomiting, dehydration, hepatomegaly, truncal hypotonia with limb hypertonia, cerebral edema, coma and multiorgan failure and death after first month of life.

- B12-responsive phenotype: it usually present in first year of life but can rarely be symptomatic neonatal age. Usual manifestation includes anorexia, failure to thrive, growth retardation, truncal hypotonia, and neuro-developmental delay.

- Adult methylmalonic acidemia: It usually manifests in adulthood with secondary complication including developmental delay; renal failure; neurological movement disorder due to basal ganglia involvement like choreoathetosis, dystonia, and para/quadriparesis; pancreatitis, growth retardation, recurrent infections because of immune impairment and optic nerve atrophy.

Diagnosis is confirmed with elevated level of methylmalonic and methyl citric acid in urine, and increased level of glycine and propionylcarinitine (C3) in serum ${ }^{6}$. Molecular Genetic Testing includes genes namely MUT, MMAA, MMAB, MCEE, and MMADHC are the genes currently known to be associated with $\mathrm{MMA}^{7}$. The MRI findings of MMA are varied and includes ventricular dilation, cortical atrophy periventricular white matter abnormality, thinning of the corpus callosum, subcortical white matter abnormality, cerebellar atrophy, basal ganglionic calcification, periventricular leucomalacia and myelination delay ${ }^{8}$. Treatment involves supportive care, stopping protein in diet, calories in diet provided through dextrose and lipid, supplementation with carnitine, vitamin B12. Emergent treatment, including dialysis to quickly remove those toxins, including MMA and ammonia. $\mathrm{N}$-carbamylglutamate may also be considered if available. In chronic treatment dietary intake includes protein diet which is low in isoleucine, methionine, threonine and valine amino acid content ${ }^{7}$.

Karimzadeh et al studied 20 patients of MMA over duration of 2002 to 2012 . In their study majority of patients $(80 \%)$ were products of consanguineous marriages. The various symptoms of the patients were developmental delay or regression (85\%), failure to thrive $(50 \%)$ and refractory seizure (20\%). MRI findings of these babies included brain atrophy, basal ganglia involvement (often in putamen), and periventricular leucomalacia 6 .
In non consanguineous families with no prior history of autosomal recessive disorder, the risk of serious congenital and genetic disorder is around 2.0 $-2.5 \%$ whereas it increases to $4.0-4.5 \%$ in children of first degree cousins.

In our experience at Fernandez hospital, out of 379 genetic disorders which were identified in infants about $84(22.1 \%)$ of them had autosomal recessive disorders. Out of these 84 children who had autosomal recessive disorders $53(63.1 \%)$ were born to consanguineous couples and $36.9 \%$ were born to non-consanguineous couples.

In our case report, Mrs. $\mathrm{R}$ had three children with MMA with her first husband, though it was a non-consanguineous marriage. During her fourth pregnancy with second husband which was again a non-consanguineous, prenatal diagnosis revealed that fetus is carrier for both MMA and Short chain acyl CO A dehydrogenase deficiency. This provides an evidence to prove that rare autosomal recessive disorders are not uncommon in non-consanguineous couples.

\section{Conclusions}

Proper diagnosis in the index child is possible with suspicion and appropriate work up. May be post-natal work up in their first child followed by an appropriate prenatal diagnosis in the mother would have prevented a marital break-up and its social implications. Also identification of two disorders in the fourth pregnancy is evidence to the belief that autosomal recessive disorders are not uncommon in our population.

\section{Learning Points/Take Home Messages}

1. Index case workup is essential to offer counselling, predict recurrence risk and also to offer prenatal diagnosis.

2. Autosomal recessive disorders are not uncommon in non-consanguineous couples and suspicion should be kept high if a couple have recurrent unexplained neonatal or fetal death.

3. Extensive counselling is needed in cases of genetic disorders because these conditions have serious implications on relationships of the couples and when these situations are not handled well it can lead to very serious consequences.

\section{References}

1. Mahoney MJ, Bick D. Acta Paediatr Scand 1987; 76(5):689-96. 
2. Oberholzer VG, Levin B, Burgess EA et al. Methylmalonic aciduria. An inborn error of metabolism leading to chronic metabolic acidosis. Arch Dis Child 1967; 42:492-504.

3. Stokke O, Eldjarn L, Norum KR et al. Methylmalonic acidemia: A newborn error of metabolism which may cause fatal acidosis in the neonatal period. Scand J Clin Lab Invest 1967; 20:313-28.

4. Rosenberg LE, Lilljeqvist AC, Hsia YE. Methylmalonic aciduria. An inborn error leading to metabolic acidosis, long-chain ketonuria and intermittent hyperglycinemia. N Engl J Med 1968; 278:1319-22.

5. Tanpaiboon P. Methylmalonic acidemia (MMA). Mol Genet Metab. 2005; 85 (1):2-6.
6. Karimzadeh P, Jafari N, Ahmad Abadi F et al. Methylmalonic acidemia: diagnosis and neuroimaging findings of this neurometabolic disorder (an Iranian paediatric case series). Iran J Child Neurol 2013;7(3):63-6.

7. Chandler RJ, Venditti CP. Genetic and genomic systems to study methylmalonic acidemia. Mol Genet Metab 2005;86:34-43.

8. Radmanesh A, Zaman T, Ghanaati H. Methylmalonic acidemia: brain imaging findings in 52 children and a review of the literature. Pediatr Radiol 2008;38:1054-61. 